\title{
Disscussion
}

\section{Alzheimer Research Forum Live Discussion: Not Dead Yet: Estrogen Deserves Another Chance ${ }^{1}$}

\author{
http://www.alzforum.org/res/for/journal/transcript.asp?liveID=44
}

\begin{abstract}
Participants: Gabrielle Strobel (Alzheimer Research Forum), Mark Smith (Case Western Reserve University), Sam Gandy (Farber Institute, Philadelphia), John Breitner (Seattle VA and University of Washington, Seattle), Phyllis Wise (University of Washington), Dominique Toran-Allerand (Columbia University), Craig Atwood (Department of Medicine, University of Wisconsin, Madison), Chris Gregory (Voyager Pharmaceutical Corporation), Rena Li (Sun Health Research Institute, Arizona), Mary McAsey (Southern Illinois University School of Medicine, Springfield), Robbie Brinton (University of Southern California), Jimmy Barbee (Voyager Pharmaceutical Corporation), Robert Struble (SIU School of Medicine AD Center), Peter Zandi (Johns Hopkins Bloomberg School of Public Health), Liqin Zhao (University of Southern California), Nancy Emerson Lombardo (Boston University School of Medicine), Jon Nilsen (University of Southern California).
\end{abstract}

Gabrielle Strobel: Welcome, everyone. I am Gabrielle Strobel, managing editor of the Alzheimer Research Forum. I am nominally the moderator today, but will stay in back to let our experienced leaders run the show.

Sam Gandy: I will get us started then.

Gabrielle Strobel: Given the ups and downs that hormone replacement therapy (HRT) has gone through, would the panelists get this going by stating briefly their current opinion on its value and prospects?

Sam Gandy: Interestingly, the Science Times section of The New York Times featured HRT this week (The New York Times, Science Times Section, 31 January 2006). Very timely for us.

\footnotetext{
${ }^{1}$ Note: Transcript has been edited for clarity and accuracy.
}

Craig Atwood: Sam, what type of HRT - conjugated equine estrogens or $17 \beta$-estradiol?

Sam Gandy: Craig, I am not sure that HRT versus estrogen replacement therapy (ERT) was broken out.

Craig Atwood: My point being that estrogens extracted from horse urine are different from human serum estrogens. Likewise, medroxyprogesterone has different effects than progesterone.

Phyllis Wise: Exactly; there are over 30 compounds in the conjugated equine estrogen (CEE) preparation.

Sam Gandy: Craig, when epidemiology is discussed, I assume CEE (Premarin) unless stated otherwise.

Nancy Emerson Lombardo: The form of ERT in the Women's Health Initiative (WHI) studies was derived from mare's urine and goes through the liver. The 
results are not a true replacement of human estrogen hormones, which plant (soy)-based products applied as topical creams (which do not go to the liver) mimic better.

Phyllis Wise: Premarin is not the only preparation used in these studies. Some used ethinyl estradiol and others used yet other preparations.

Gabrielle Strobel: Also surprising in that New York Times story (The New York Times, Science Times Section, 31 January 2006) was that prescription rates have not plummeted as far as one would think following the WHI data. The piece also did not deal with AD but mostly cardiovascular disease. It did quote an opponent of HRT (a cardiology professor) as saying that people who still study it are simply unwilling to let go of a cherished hypothesis, regardless of the data.

John Breitner: I am fairly amazed, because I sense a reversal in the common view that because a trial negates observational data, the latter are faulty. The issue of timing seems to be in everyone's mind. How did that happen?

Phyllis Wise: As basic scientists, we have followed the WHI work with great interest. We have been focusing our recent studies on deciphering the reasons for discrepancies between it and other studies.

Gabrielle Strobel: Phyllis, can you make some explanations for those discrepancies, based on your work?

Phyllis Wise: I think that timing, dose, and formulation are critical differentiators of the response. For example, the average age of the women in the WHI was 61 , and they had not received prior hormone therapy (HT). CEE clearly has different effects than $17 \beta$ estradiol. Medroxyprogesterone acetate binds to both the progesterone receptor and the androgen receptor.

Mark Smith: Phyllis, how representative of HRT users were the women in the WHI study? My feeling is they were not very representative.

John Breitner: Mark, I do not think we should be distracted by the ability to generalize the study population when it comes to trials. Internal validity is virtually all any trial can promise.

Dominique Toran-Allerand: I think it is far more than that the trials were started too late. All of the
WHI studies were flawed because of failure to take into account the biology of estrogens and of the estrogen receptors.

Gabrielle Strobel: Dominique, very interesting; could you expand a bit for us?

Dominique Toran-Allerand: Gabrielle, comments have already been made on the differences between ovarian $17 \beta$-estradiol and CEE, but equally important is the fact that in the WHI studies the hormones, CEE and Provera (medroxyprogesterone acetate), were given for years without interruption. Unlike with many compounds which increase the levels of their receptors, prolonged exposure to estrogens decreases the levels of the estrogen receptor so that with time, the cells become much less responsive to estrogens. Moreover, by giving estrogens and progestins concurrently instead of the physiological manner which is sequential, estrogen is unable to up-regulate the progestin receptor to enable it to respond to progesterone, as it does after ovulation (one of its important functions), and progestins are unable to down-regulate the estrogen receptor - one of progestin's important functions - so neither hormone can act physiologically.

Gabrielle Strobel: Dominique, very interesting. So future trials should use estrogen breaks?

Dominique Toran-Allerand: Gabrielle, absolutely. That is the way the body is normally exposed to these hormones during the reproductive period.

Sam Gandy: I agree with Dominique's point about the cyclical nature of hormones and the absence thereof in clinical trials.

Craig Atwood: Dominique, Sam, however, there is no cycling of estrogen and progesterone during pregnancy, or during lactational amenorrhea? So is cycling that important?

Dominique Toran-Allerand: Craig, while there may be no cycling of estrogen and progesterone during pregnancy, human gestation is finite ( 9 months) and does not extend for years as in the WHI trials.

Craig Atwood: Dominique, but if you take a women who has five children (almost 4 years of pregnancy) and lactation amenorrhea for $\sim 3$ years each ( 15 years), that is almost 20 years of no cycling. And this is the 
normal hunter-gatherer situation, from which we have evolved recently.

Phyllis Wise: Dominique, we have had several opportunities to talk about this, but just for the record: Downregulation of estrogen receptor (ER) occurs when pharmacological levels of hormone treatment are used. We have shown that with our very low hormone treatments, the receptors remain. To me, this is a very significant point, because if we can use low levels of estrogen and still get protection, it may be important.

Gabrielle Strobel: Phyllis, are birth control pills with the lowest doses used to date in the range you just mentioned?

Phyllis Wise: Gabrielle, contraceptive pills still use relatively high doses because you have to prevent ovulation or implantation. The whole question is whether in postmenopausal women, where it is not necessary to control fertility, you can use much lower doses and still get the protective actions. We believe you can.

Dominique Toran-Allerand: Phyllis, we have found that continuous proestrus levels of estrogen given to mice (which are really not pharmacological) do elicit down-regulation of the estrogen receptor if given for many months without interruption.

Phyllis Wise: Dominique, I agree. Proestrus levels should be considered high and could down-regulate receptors. Our paradigm has used replacement levels that are characteristic of the other days of the cycle.

Sam Gandy: The main point of the Times piece was that all fields are revisiting HRT with the notion that the clinical trials that have turned us off were all begun too late. The issue now is, do we have to redo everything and start perimenopausally? How will that impact bone, heart, vessels? Brain?

Rena Li: How about the dosage of ERT?

Chris Gregory: There are several issues around ERT that remain interesting to us. In particular, we wonder whether in the ancillary WHI Memory Study (WHIMS), findings relate to the inability of estrogen to down-regulate luteinizing hormone (LH) levels when there has been time (years) between the onset of menopause and the administration of estrogen. In other words, does the prolonged absence of estrogen prior to ERT dysregulate the hypothalamus-pituitarygonadotrophin (HPG) axis, leading to the inability of estrogen to turn off local/systemic LH production?

Mark Smith: Chris, estrogen does not regulate LH after several years postmenopause (or ovariectomy in animal models).

Phyllis Wise: Mark, my understanding is that estrogens will continue to feed back negatively even after an animal has been ovariectomized for a prolonged period of time.

Craig Atwood: Phyllis, that is correct.

Mark Smith: Phyllis, I have read in several papers otherwise. . . we should discuss.

Sam Gandy: I was surprised to learn this week that there is an NIH early estrogen study that has recently begun. I do not recall the acronym. I thought that the Kronos Early Estrogen Prevention Study (KEEPS) was the only thing going.

Jon Nilsen: Sam, are you thinking of the Early Versus Late Intervention Trial With Estradiol (ELITE) run by Howard Hodis, testing the effect of early versus late HRT on the cardiovascular system?

Sam Gandy: ELITE is the one; yes, thanks. Is there a cognitive component to ELITE, Jon?

Jon Nilsen: Sam, there is a secondary outcome measure of neurocognitive function proposed for ELITE.

Sam Gandy: Phyllis, can you tell us anything about KEEPS? Will there be periodic interim analyses?

Phyllis Wise: Sam, my understanding is that the trial will use CEE compared to estradiol in patch form supplemented with progesterone, and that endpoints will be measured at annual intervals. But do not quote me on this.

Gabrielle Strobel: Phyllis, in KEEPS, what are the endpoints? Just clinical (dementia diagnosis, cognitive performance, etc.), or is there also blood and CSF work on biomarkers, $\mathrm{A} \beta /$ tau levels, brain imaging?

Phyllis Wise: Gabrielle, the major endpoints are cardiovascular, but some cognitive endpoints will be mea- 
sured. Again, I feel a bit uncomfortable going into too much more detail until I consult with Mitch Harman about the final design that they decided to use. There was a lot of discussion about how much could be done with the funds and the relatively small group of women.

Gabrielle Strobel: Thanks, Phyllis. There is much excitement about biomarkers in $\mathrm{AD}$ research these days. It would be wonderful if they could be included, but I see that money is an issue, as always.

Craig Atwood: Sam, how old are the ELITE participants?

Sam Gandy: Craig, no age is stated. Just "completed menopause" as the entry criterion.

Craig Atwood: Sam, it would be better if they had started a little earlier.

Rena Li: Our preliminary data from animal studies support early treatment rather than late.

Phyllis Wise: Rena, great confirmation of the principle that time of treatment is critical.

Chris Gregory: Rena, reduced estrogen in Alzheimer's disease (AD) brains compared to normal brains with similar serum estrogen levels [1] correlated nicely with our gonadotrophin-based mechanistic hypothesis of AD.

Phyllis Wise: Rena, we too have completed a preliminary study that shows that when mice are ovariectomized and then not treated for a long period, subsequent estradiol does not protect against stroke injury. With some luck, we will submit this in a couple of months. Daniel et al. [2] show that immediate replacement in middle-aged rats improves memory but not after a long period of deprivation [2].

Rena Li: Phyllis, our data show that late treatment does not improve AD pathology in animal models.

Mark Smith: Rena, mechanistically, why do you think that is?

Rena Li: Because late estrogen treatment does not activate clearance of $\mathrm{A} \beta$.

Sam Gandy: Rena, how did your animals parse out with respect to gender? Did males and females show the same thing?
John Breitner: But who can follow immediately postmenopausal women for 30 years to observe incidence of dementia and AD? It seems to me this is one area where there is an urgent need for surrogate indicators all caveats notwithstanding.

Peter Zandi: John, right. I wonder how a 5-year trial, assuming this is the distance, can show anything relevant, early versus late.

Craig Atwood: John, Mark Sagar has a study here in Wisconsin - the Wisconsin Registry for Alzheimer's Prevention (WRAP) - that is doing just that. The study has recruited over 600 individuals, who must have a parent with confirmed AD. I am not sure how many are on hormone replacement.

Nancy Emerson Lombardo: John and Mark, brain healthy nutrition and exercise will help you stay alive with no AD a lot longer than NSAIDs.

Craig Atwood: John, better still, take leuprolide. Steve Austad showed that leuprolide increased longevity in rats by 18 percent with 6 months of treatment.

Sam Gandy: John, is 30 years the real minimum number of years to have enough power to see an effect, based on the average age at onset of menopause and $\mathrm{AD}$ ?

John Breitner: Sam, as usual, it all comes down to money and resources. The younger the cohort, the more subjects you need to achieve the number of incident cases, which is what drives power.

Gabrielle Strobel: John and Peter, this brings up an interesting question: What sorts of shorter, cheaper trials can address this time factor (mid-life treatment versus late-life diagnosis)? That would be worth brainstorming about, it seems. Everyone, can animal models be employed to address this question?

John Breitner: Gabrielle, animal models are clearly a contender, but the problem is, we do not know how true to life the animal models are. Most of our models focus squarely on the $\mathrm{A} \beta$ hypothesis. Robbie Brinton's work suggests there may be a timing interplay between $\mathrm{A} \beta$ and estrogen treatment, but I think I am more intrigued with the biomarkers idea. If only we could get biomarkers for $\mathrm{AD}$, such as the CD4 counts and viral 
load we have for HIV disease! But that would probably require that we know a lot more about root causes.

Sam Gandy: John, Pittsburgh compound B (PIB).

John Breitner: Sam, yes. Possibly PIB. It is amazing also how little we know about the longitudinal trace of some of the lower-tech biomarkers in preclinical phases of $\mathrm{AD}$.

Sam Gandy: Gabrielle, there are some interesting strategies to try to cut the cost of long trials by using phone screening.

Gabrielle Strobel: Please expand, Sam. Is testing people for inclusion/exclusion criteria a major cost factor?

Sam Gandy: Basically, versions of the various cognitive instruments are being adapted for use by phone, so that clinic visit expense is eliminated.

Gabrielle Strobel: John, very interesting. I am just putting finishing touches on a meeting report on an academic/biotech/pharma workshop on biomarkers for AD. Tony Wyss-Coray's talk on his proteomic fingerprinting test of a composite of 12 inflammation-related markers was a highlight. Small subject numbers, but it predicted with 97 percent accuracy.

Sam Gandy: John, can some assumptions be made in order to guesstimate a plausible prediction regarding duration and reasonable cohort sizes, etc.?

John Breitner: Yes, Sam, but the results are likely to be disheartening. Especially in the present funding environment, I think animal models or especially surrogate indicators are the way to go.

Sam Gandy: John, but the FDA will want humans before giving its blessing, no?

John Breitner: Sam, almost certainly yes.

Chris Gregory: John, as imaging and biomarker analysis and genomics (proteomics and metabolomics) continue to be increasingly applied to AD research, there will hopefully be an enhanced ability to identify patients likely to progress and thus shorten the length of those trials and decrease the number of patients necessary for the trial.
Rena Li: Monitoring hormone levels is important. I know the NIH is calling for small business grants to develop small medical devices for that purpose.

Robert Struble: I think, before we focus, we should emphasize Dr. Toran-Allerand's earlier statement (and it is showing up again and again). We need to understand the biology of the nervous system as it relates to hormone therapy. As we have seen, clinical intervention studies not based on good science can cause major problems.

Gabrielle Strobel: All, what would be some good biomarkers to develop to track the effect of estrogen?

John Breitner: Gabrielle, we know almost nothing about which putative AD biomarkers are sensitive to change in preclinical stages of $\mathrm{AD}$ pathogenesis.

Sam Gandy: All, does the complexity of hormones, coupled with the chronic nature of Alzheimer's, equate to a hopelessly complex situation?

Craig Atwood: Do high estrogen levels during pregnancy lead to high progesterone receptor (PR) levels? Or does the high progesterone suppress PR level?

Sam Gandy: What feasible model for Alzheimer's would everyone agree would be the best preclinical surrogate for human disease? Transgenic monkeys?

Mark Smith: Sam, Down syndrome (DS).

Gabrielle Strobel: Sam, is that a feasible model?

Sam Gandy: Mark, there are no cognitive norms for DS, and every DS person is slightly different. I cannot see any enthusiasm for DS.

Mark Smith: Sam, but it is the best model.

Gabrielle Strobel: Mark, can you imagine the ethical/informed consent/regulatory issues with experimenting on DS patients with compounds shown to increase the risk of cardiovascular disease?

Mark Smith: Gabrielle, complex, I agree, but no more so than testing drugs in AD patients. Has anyone looked at HRT in Down syndrome?

Sam Gandy: Nicole Schupf. 
John Breitner: Sam, what does Nicole find?

Sam Gandy: Age at onset of dementia in DS correlates with menopause [3].

Nancy Emerson Lombardo: Mark et al., I also think Down's could be the way to go. One could select a narrowed population with particular criteria. Is there any indication the Down's research community is open to estrogen therapy?

John Breitner: Nancy, Down's study would be very interesting, but probably would not pass muster for clinical use because of the generalizability issue.

Nancy Emerson Lombardo: John, good point, though it might give us a quicker understanding of mechanisms that could be applied to the more usual population, and by then we might have the biomarkers to measure changes in the brain presymptomatic to AD.

Gabrielle Strobel: All, has funding for estrogen-brain research dropped further in the wake of the WHI disappointment?

Craig Atwood: Sam, Gabrielle, a big yes. It is surprising to me that in Europe, $17 \beta$-estradiol is used for HRT rather than CEEs, and most studies from Europe suggest that this form of HRT is beneficial on a number of levels. Any comments?

Liqin Zhao: Yes, Craig, we believe the formulation of estrogen therapy is another very crucial factor affecting study outcomes.

Nancy Emerson Lombardo: Estradiol is what we should be using in the US as well.

John Breitner: All, longitudinal biomarker data could at least guide us as to which HRT formulations to test in long-term human trials.

Liqin Zhao: From our previous in vitro studies we have found that only select estrogen components contained within the complex formulation of CEE are effective at the levels they attain in plasma.

Sam Gandy: And now there is Ellis Levin's endoplasmic reticulum estrogen receptor (ERER) as well [4, $5]$.
Gabrielle Strobel: Sam, where does that fit in? What hypothesis does it suggest? I cannot picture it. . .

Sam Gandy: The ERER plays a role in calcium homeostasis, which certainly could dovetail with conventional models of neurodegeneration.

Chris Gregory: Phyllis, are the brain estrogen levels (versus serum levels) more important for protective effects?

Phyllis Wise: Chris, Dominique's data would suggest that brain estrogen levels are important, and there may be different estradiol isomers in the brain than in plasma.

Rena Li: The brain does make estrogen itself and has brain-specific aromatase [6].

Dominique Toran-Allerand: Regarding brain versus serum estrogen levels, we have found by mass spectrometry that the level of endogenous $17 \beta$-estradiol levels in the neocortex, hippocampus, and cerebellum are very, very low, but the levels of $17 \alpha$-estradiol, which does not circulate and which appears to be made in the brain, are astronomical and likely to be very important.

Gabrielle Strobel: Rena, does this brain-specific estrogen production change after menopause? Are the ovaries and the brain production coupled?

Rena Li: Gabrielle, that is not clear.

Phyllis Wise: Rena, I think that we should keep in mind that aromatase knockout mice will have higherthan-normal levels of androgens (since they cannot be converted to estradiol) and that androgens themselves may have influence. In stroke models, most investigators have found that androgens exacerbate. Christian Pike has shown that, in vitro, androgens may protect against amyloid- $\beta$ injury $[7,8]$.

Rena Li: Phyllis, that is true in our animals. Male animals do not develop the same degree of pathology as female aromatase knockouts.

Chris Gregory: Rena, Dominique, are we not talking about "autocrine" estrogen signaling in the brain?

Rena Li: Chris, we really do not know. We are working on it. 
Chris Gregory: Rena, have you considered that brain estrogen levels may influence brain gonadotrophin levels (e.g., LH)?

Rena Li: Chris, good point and should be further pursued, but we have not done it yet.

Craig Atwood: A lot of talk about the sex steroids, but there is a Phase III trial ongoing for leuprolide acetate. Since leuprolide suppresses sex steroid production, and the Phase II results look very encouraging, that is, no cognitive loss over 48 weeks, any thoughts/comments on other hormones that might be involved, or other mechanisms? Chris, do you wish to discuss?

Sam Gandy: Craig, I gather that those men get supplemental testosterone.

Gabrielle Strobel: Craig and Chris, good point. Can you enlighten me about the discrepant findings of leuprolide effect between your/Voyager studies and work by Sam and the Finnish study by Salminen [9]?

Craig Atwood: In the women's Phase II, women did not get estrogen add-back. In a second Phase II (men only), the men are being supplemented with testosterone. This is ongoing. Results will be available midyear, I believe.

Chris Gregory: Craig, Sam, the males in Voyager's Phase II studies are receiving supplemental testosterone (T) to maintain their T levels. We think that this is a key distinction with the Finnish study.

Mark Smith: Lupron was given based on the gonadotrophin hypothesis. Leuprolide acetate also works very, very well in $\mathrm{Tg} 2576$ mice [10].

Chris Gregory: Mark, could you comment on the effects you see with leuprolide in $\mathrm{Tg} 2576$ s?

Mary McAsey: Craig, in the women's Phase II study, was there an increase in depression rates in women on lupron?

Craig Atwood: Mary, not that I am aware of. Chris?

Chris Gregory: Mary, there were no negative effects on depression in the women's study.

Craig Atwood: Obviously if leuprolide suppresses sex steroids, and cognition is not declining, do we need to look elsewhere for an etiological agent that drives the disease? I should mention that those taking leuprolide were also taking acetylcholinesterase inhibitors.

Chris Gregory: Craig, as you know, leuprolide appears to maintain cognition and lower amyloid- $\beta$ levels.

Phyllis Wise: Craig and Mary, were estradiol levels measured in these studies.

Craig Atwood: Yes, but as you know, leuprolide suppresses estrogen to castrate levels.

Gabrielle Strobel: Eef Hogervorst posted a comment on the Alzheimer Research Forum suggesting the estrogen effect in aging women lasts 2-3 months. Any thoughts?

Sam Gandy: That sounds like a mood or attention effect.

Rena Li: Anybody know about phytoestrogen trials? Are there any?

Craig Atwood: Carey Gleason at University of Wisconsin-Madison has some interesting phytoestrogen trials happening.

Sam Gandy: I think that all we have done is identify the problems. Anyone have any serious hope that anything truly positive will come out of all this in our lifetimes?

Mark Smith: Sam, for transgenic mice the prospects have never been better.

Nancy Emerson Lombardo: Sam, in the broader sense of finding out what we can do in our forties, fifties, and sixties to reduce the risk of $\mathrm{AD}$ and understand its true (complicated) etiology-yes, yes, yes. But it will not be just estrogen or just insulin. . . or just any one factor, I would guess.

John Breitner: All, there are more or less two lines of chat here. One on pathophysiology and modeling, the other on development of interventions for humans and the problems faced here. I think we need to pursue both. The lab scientists can tell us more about which interventions could be used, and when, while those involved in clinical trials and human studies should be thinking about how most effectively and efficiently to test these interventions. 
Robert Struble: We seem to be losing focus on the important question of protecting from dementia and focusing on mechanisms. The key question is whether there is a pattern/method to use $17 \beta$-estradiol (E2) as a "neuroprotective" agent. If we find it is neuroprotective, then let us find out why. The mechanism is irrelevant at the moment when women (and men) are aging. Rodent studies show that after ovariectomization, E2 improves performance or protects from stroke, but long-term or delayed treatment loses efficacy. Why? What can we do to improve protection and can that be translated to clinical trials?

Sam Gandy: I guess that we can all hope that KEEPS or ELITE raises enthusiasm for continuing to hammer away. Does anyone know when we can expect to hear updates from KEEPS or ELITE?

Robbie Brinton: The ELITE trial is being conducted by Howard Hodis here at University of Southern California. It will be quite a while before data from the ELITE trial will be available; recruitment is still in process, but remarkably, the recruitment process is going well.

Sam Gandy: Robbie, how long is quite a while regarding ELITE? Five years? More?

Robbie Brinton: Sam, I would guess that the ELITE trial is 5 years (funding cycle), but I will check with Howard. There are multiple endpoints including a supplement for cognition which is being conducted by Victor Henderson.

Sam Gandy: There is a bit of a circular problem in that the basic science funding is suffering because of the state of the clinical trial data.

Rena Li: To all, start ERT studies at younger ages.

Phyllis Wise: Sam, absolutely. I think that a great deal more basic science research should be steered to looking at the importance of timing, preparation, and dose. Once we figure out the mechanisms of action that are involved in protective pathways, we should be able to design better selective estrogen receptor modulators (SERMs). Although it would be ideal to measure risk of $\mathrm{AD}$, that may be prohibitively expensive. If we can use other endpoints that appear earlier and are good indicators, we may be able to design better clinical studies.
Rena Li: I think dosage is relevant because each individual will have a different level of estrogen due to absorption rate variability. Therefore, monitoring estrogen level in the ERT user might be very important.

Robbie Brinton: All, our mechanistic analyses indicate that convergence of E2 action onto the mitochondria is a critical factor in regulating calcium homeostasis. Our current studies are determining the impact of E2 and progestins on mitochondrial calcium buffering capability prior to, versus following, exposure to disruptions in calcium homeostasis. The data thus far are quite interesting. A question to the community, determining the key biomarkers for prevention remains unanswered in my mind. Ideas?

Sam Gandy: Robbie, my answer is PIB.

Chris Gregory: We think PIB might be a great tool.

Sam Gandy: Serial PIB scans every year after menopause - / + ERT?

Chris Gregory: Sam, agreed, that could be a very interesting study, using PIB scans every year after menopause $(-/+$ ERT $=+/-$ LH $)$.

Nancy Emerson Lombardo: Sam, what is the going price per scan to use PIB as a biomarker in a clinical trial?

Sam Gandy: Nancy, I do not know. Outrageous, I am told, but I do not know the figure. I gather that General Electric (GE) has the rights and has priced it out of everyone's reach. But that is gossip. I do not know that on authority.

Dominique Toran-Allerand: I think we need to look more at the non-ovarian $17 \alpha$-estradiol endogenous to the brain, as a potential therapeutic tool for menopause.

Sam Gandy: Dominique, are those available for clinical trials or only basic science?

Dominique Toran-Allerand: Unfortunately, I think only for basic science now.

Jon Nilsen: Sam and Dominique, $17 \alpha$-estrodiol has been used in Phase I clinical trials [11].

Dominique Toran-Allerand: Jon, what dosage and how was it administered? I think it needs to be given transdermally, not orally since it does not circulate. 
Jon Nilsen: Dominique, I do not know the dosage, but it was administered as sodium sulfate conjugate orally.

Jimmy Barbee: The Alzheimer Disease Neuroimaging Initiative (ADNI) biomarkers arm may provide much insight into the prevention arena, as will the imaging side.

Mark Smith: Last question, Sam: What is a good argument against the Voyager Phase II results?

Sam Gandy: Mark, I simply want to see a definitive trial. I agree that the data are encouraging, but the sample size ("n") is small. Trials with small n's (like experiments with same) are often misleading. I say the exact same thing about statins. And HRT, for that matter.

Mark Smith: One and a half million is bigger than most of my experimental n's.

Sam Gandy: Epidemiology is not the gold standard, Mark. Epidemiology can be misleading, too. HRT is based on epidemiology. Sufficiently powered, randomized, double-blind, placebo-controlled trials are required.

Nancy Emerson Lombardo: Agreed, but by the time all the clinical trials and prevention trials are funded and completed, the baby boom generation will all be dead or demented. So what do we do in the meantime? We keep plugging along and doing the best we can to prove with gold standard random controlled trials, but in the meantime we have a public health crisis that needs addressing, which the Alzheimer's Association and now the Centers for Disease Control (CDC) are making a start with. I think we scientists need to have this on our minds as well.

Chris Gregory: Sam, we agree about the controlled clinical trials and that is what we are working on right now.

Sam Gandy: Yes, I know about Voyager's trials, but strictly speaking, "working on" is simply not "there." I am not disagreeing or criticizing. I am just withholding judgment until the data are in.

Mark Smith: Fair enough. What data does HRT have that leuprolide has to have to make it more promising?
Sam Gandy: Nothing. The clinical trials are a bust, at least perhaps because they were done too late. If a perimenopausal HRT trial is ever done and is negative, I give up.

Mark Smith: Then, as a treatment, HRT is a bust?

Sam Gandy: Absolutely. It is prevention or nothing for HRT at this point.

Chris Gregory: Sam, we are not mechanistically far apart from the discussions raised in today's forum. The well-powered trials will provide the answer, but like everyone else doing AD studies, these trials are long (at least 12 months now and in some cases 18 months). Not an excuse, just the reality that we all have to deal with.

Nancy Emerson Lombardo: Sam, but be sure the perimenopausal trials are done with the right humanlike ERT and properly administered, per this discussion. If Premarin were used only, then I still would not necessarily believe the results.

Sam Gandy: Nancy, I agree that the issues of cycling, preparation, progestins, etc., are all issues that must be accounted for and optimized. It makes more sense to me to "prevent" the acute hormone withdrawal than to allow the body to re-equilibrate for 20 years and then try to go in and get anywhere.

Mark Smith: But as John said, prevention is very expensive and fraught with its own problems.

Sam Gandy: Mark, that is why these phone instruments are being developed, to cut the cost of prevention trials. Just because it is hard does not mean we should not try...

Nancy Emerson Lombardo: Sam, I totally agree. By the way, probably the correct administration of a phytoestrogen or phytoHRT would be transdermally; that would also prevent the heart problems according to the HRT specialists I follow. They predicted the WHI MS trial would fail and that Premarin creates heart/stroke problems.

Sam Gandy: Phytoestrogens are another variable in the queue as far as I am aware. We have reached the end of our hour. Thank you all very much for this interesting discussion. 


\section{References}

[1] X. Yue, M. Lu, T. Lancaster, P. Cao, S. Honda, M. Staufenbiel, N. Harada, Z. Zhong, Y. Shen and R. Li, Brain estrogen deficiency accelerates Abeta plaque formation in an Alzheimer's disease animal model, Proc Natl Acad Sci USA 102 (2005), 19198-19203.

[2] J.M. Daniel, J.L. Hulst and J.L. Berbling, Estradiol replacement enhances working memory in middle-aged rats when initiated immediately after ovariectomy but not after a longterm period of ovarian hormone deprivation, Endocrinology 147 (2006), 607-614.

[3] N. Schupf, D. Pang, B.N. Patel, W. Silverman, R. Schubert, F. Lai, J.K. Kline, Y. Stern, M. Ferin, B. Tycko and R. Mayeux, Onset of dementia is associated with age at menopause in women with Down's syndrome, Ann Neurol 54 (2003), 433438.

[4] C.M. Revankar, D.F. Cimino, L.A. Sklar, J.B. Arterburn and E.R. Prossnitz, A transmembrane intracellular estrogen receptor mediates rapid cell signaling, Science 307 (2005), 16251630.

[5] R.J. Pietras, E.R. Levin and C.M. Szego, Estrogen receptors and cell signaling, Science 310 (2005), 51-53; author reply 51-53.
[6] H. Sasano, K. Takashashi, F. Satoh, H. Nagura and N. Harada, Aromatase in the human central nervous system, Clin Endocrinol (Oxf) 48 (1998), 325-329.

[7] C.J.Pike, Testosterone attenuates beta-amyloid toxicity in cultured hippocampal neurons, Brain Res 919 (2001), 160-165.

[8] M. Ramsden, A.C. Nyborg, M.P. Murphy, L. Chang, F.Z. Stanczyk, T.E. Golde and C.J. Pike, Androgens modulate betaamyloid levels in male rat brain, $J$ Neurochem 87 (2003), 1052-1055.

[9] E.K. Salminen, R.I. Portin, A.I. Koskinen, H.Y. Helenius and M.J. Nurmi, Estradiol and cognition during androgen deprivation in men with prostate carcinoma, Cancer 103 (2005), 1381-1387.

[10] G. Casadesus, K.M. Webber, C.S. Atwood, M.A. Pappolla, G. Perry, R.L. Bowen and M.A. Smith, Luteinizing hormone modulates cognition and amyloid-beta deposition in Alzheimer APP transgenic mice, Biochim Biophys Acta 1762 (2006), 447-452.

[11] J.A. Dykens, W.H. Moos and N. Howell, Development of $17\{$ alpha\}-Estradiol as a Neuroprotective Therapeutic Agent: Rationale and Results from a Phase I Clinical Study, Ann N Y Acad Sci 1052 (2005), 116-135. 